\title{
Métrica para Avaliação Quantitativa do Nível de Inversão de Ordem de Pacotes Ópticos e Rajadas em Redes Roteadas por Deflexão
}

\author{
Luiz Henrique Bonani, Felipe Rudge Barbosa, Rangel Arthur, Edson Moschim
}

\begin{abstract}
Resumo-Em redes ópticas comutadas por pacotes e roteadas por deflexão, freqüentemente verifica-se que em certos níveis de carga na rede muitos dos pacotes sofrem inversão de ordem na chegada a seus respectivos destinos. Desta forma, este trabalho apresenta a proposta de uma métrica para avaliação quantitativa desse nível de desordem. Basicamente, faz-se uso da estatística de distribuição de tráfego para modelar o comportamento da distribuição dos tempos de atraso das chegadas e, com isso, estabelecer um parâmetro de correlação com o comportamento observado efetivamente na rede. Os primeiros resultados mostram-se bastante promissores e pretende-se aperfeiçoar a técnica com outras formulações.

Palavras Chave-Ordenação de Pacotes, Redes Ópticas Comutadas por Pacotes, Roteamento por Deflexão.
\end{abstract}

Abstract-In Optical Packet and Burst Switching (OPS/OBS) networks using deflection routing, frequently we see that with certain levels of network load, many of received packets suffer inversion of order in the arrival at their destinations. This work presents a proposal for a metric to be used in the quantitative evaluation of disorder level. Basically, we use the statistical distribution of traffic to model the behavior of delay distribution of arriving packets and establish a correlation parameter with the behavior effectively observed in the network. The first results are satisfactory e we intend to improve the technique with other formulations.

Index Terms-Inversion of Order, Optical Packet Switched Networks, Deflection Routing.

\section{INTRODUÇÃO}

Já é realidade que as redes ópticas de nova geração se constituem hoje do principal veículo para o tráfego de grandes quantidades de informação. Por conta disso, novos paradigmas de transmissão têm sido apresentados, incluindo a utilização da comutação por pacotes e por rajadas na camada óptica [1][2][3]. Apesar de esta não ser a realidade atual, é hoje a saída mais razoável para a obtenção de granularidade e escalabilidade em redes ópticas. Da mesma forma, o custo tecnológico de implementação desse tipo de rede ainda é muito alto e soluções simples, como a utilização de topologias em

L. H. Bonani e R. Arthur, Centro Superior de Educação Tecnológica, Universidade Estadual de Campinas (Unicamp), Limeiras-SP - Brasil, \{bonani,rangel\}@ceset.unicamp.br, F: +55-19-21133492.

F. R. Barbosa e E. Moschim, Faculdade de Engenharia Elétrica e de Computação, Universidade Estadual de Campinas (Unicamp), Campinas-SP - Brasil, \{rudge, moschim\}@dsif.fee.unicamp.br, F: +5519-37883766. malha com configuração 2x2 (dois enlaces de entrada e dois de saída) e utilização de roteamento por deflexão (DR) para resolução de contenda entre rajadas ou pacotes ópticos tornam-se uma opção bastante atrativa.

Contudo, um problema importante em redes ópticas comutadas por pacotes ou rajadas, quando submetidas ao roteamento por deflexão, é que estas podem apresentar níveis de desordem relativamente altos [4] em seus nós de destino sob certas condições de tráfego. Esses níveis de desordem são prejudiciais no destino, pois desta forma necessita-se de um tempo adicional para o reordenamento dos pacotes através de um armazenador de saída, além do armazenador de entrada. Portanto, apresenta-se no presente trabalho uma métrica para avaliação quantitativa desse nível de desordem, fazendo-se uso da estatística de distribuição de tráfego para modelar o comportamento da distribuição dos tempos de atraso das chegadas e, com isso, estabelecer um parâmetro de correlação com o comportamento observado efetivamente na rede.

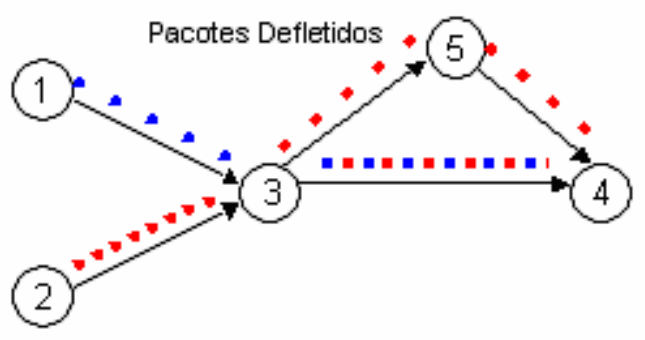

Fig. 1. Exemplo de pacotes fora de ordem numa rede óptica com DR.

Para ilustrar como o problema ocorre, apresenta-se na Fig. 1 um exemplo segundo o qual o fluxo de pacotes proveniente do nó 1 , com destino ao nó 4 , possui uma taxa de bits que é aproximadamente a metade da taxa do fluxo iniciado no nó 2. Considerando aqui que haja colisões entre os dois fluxos de pacotes no nó 3, como há resolução de contenda por deflexão alguns dos pacotes com origem no nó 2 poderão seguir pelo caminho mais curto até atingir o nó 4, devido ao fato de não haver colisões em alguns instantes de tempo. Deste modo, alguns pacotes seguem o trajeto até o nó 4 passando por 2 enlaces, enquanto outros pacotes seguirão pelo caminho alternativo, percorrendo 3 enlaces. Assim, imaginando que os pacotes sejam roteados no nó 3 , alternando o caminho até o nó 4 para os pacotes que se originam no nó 
2, então no nó 4 haverá uma inversão da ordem de chegada dos pacotes, pois um pacote chaveado de forma a alcançar o destino pelo menor caminho, que tenha sido encaminhado no nó 3 logo após um pacote que toma o caminho alternativo, deverá chegar ao nó 4, antes deste pacote que foi defletido.

\section{Modelagem do Problema}

Nesse exemplo é fácil perceber que, se todos os pacotes chegassem ao nó de destino sem inversão de ordem, o tempo de chegada $\mathrm{Y}(\mathrm{Y}=\mathrm{t} 1, \mathrm{t} 2, \mathrm{t} 3, \ldots$, tn $)$ e a ordem $\mathrm{X}$ $(\mathrm{X}=1,2,3, \ldots, \mathrm{n})$ dos pacotes estariam relacionados através de um polinômio de primeira ordem, como mostrado na Equação (1), onde $C a$ e $C b$ são respectivamente os coeficientes angular e linear de uma reta.

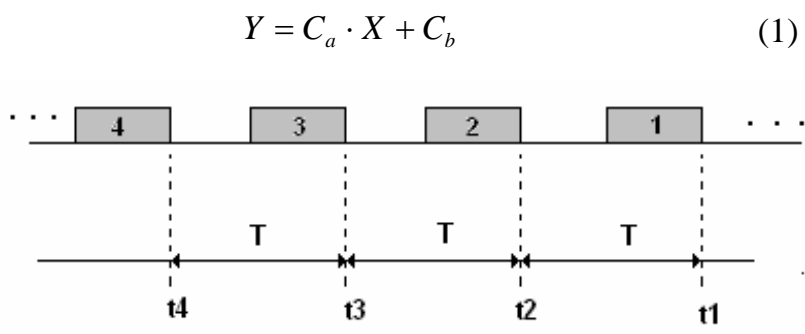

Fig. 2. Chegada de pacotes no nó de destino (caso SF).

A Fig. 2. , mostra o comportamento da chegada de pacotes para o roteamento SF (Store-and-Forward), quando todos os pacotes seguem pela mesma rota sem serem defletidos. Nesse caso, observa-se um fluxo de tráfego em que todos os pacotes chegam estritamente na mesma ordem e com a mesma distância temporal T, em que T é dado pela Equação (2)

$$
T=t_{2}-t_{1}=t_{3}-t_{2}=t_{n}-t_{n-1}
$$

O coeficiente angular $\mathrm{Ca}$ é dado pela derivada dessa função, que sendo uma reta pode ser escrita como mostrado na Equação (3).

$$
C_{a}=\frac{y_{2}-y_{1}}{x_{2}-x_{1}}=\frac{t_{2}-t_{1}}{2-1}=T
$$

O intervalo entre pacotes sucessivos T é composto pelo máximo intervalo entre pacotes $t_{\max }$, somado ao tempo do pacote, que por sua vez é dado pela Equação (4), onde $P$ é o tamanho do pacote em bits e $S$ a banda do enlace.

$$
T_{p}=\frac{P}{S}
$$

Desta forma, Ca pode ser escrito como

$$
C_{a}=T=t_{\max }+T_{p}=\frac{P}{R} \cdot\left(1-\frac{R}{S}\right)+\frac{P}{S}=\frac{P}{R}
$$

onde $R$ é a taxa de bits do fluxo de tráfego.

$$
Y=C_{a} \cdot\left(X-X_{0}\right)+Y_{0}
$$

Assim, a reta pode ser dada pela Equação (6), onde o ponto $\left(X_{0}, Y_{0}\right)$ se refere a qualquer ponto que pertença a esta. Para o caso do roteamento SF, o primeiro pacote chegará ao nó de destino no tempo necessário para percorrer todo o caminho entre nó de origem e nó de destino. Portanto, se o tempo em que o primeiro pacote que chega ao nó for conhecido $\left(Y_{0}=t i\right)$, pode-se escrever a equação da reta como

$$
Y=C_{a} \cdot(X-1)+t_{i}
$$

Isso porque $X_{0}=1$. Como o coeficiente linear $C b$ da reta é definido como o ponto de intersecção com o eixo das abscissas, pode-se escrevê-lo como descrito na Equação (8).

$$
C_{b}=Y(0)=t_{i}-C_{a}
$$

Desta forma, a reta para o caso de roteamento SF fica inteiramente caracterizada. Deve-se notar que o coeficiente angular depende somente da taxa de bits $R$ quando o tamanho dos pacotes $P$ é mantido constante. Além disso, como o coeficiente linear depende do tempo de chegada $t_{i}$, que por sua vez depende exclusivamente do comprimento do trajeto entre os nós de origem e de destino, este é um parâmetro bastante sensível ao atraso.

Além da análise apresentada, se fosse possível a aquisição de todos os tempos de chegada dos pacotes para o roteamento SF, poder-se-iam obter os mesmos valores dos coeficientes angular e linear através do cálculo da reta média entre os pontos, usando-se o método de regressão linear [5][6]. Nesse caso, como os parâmetros da reta obtida seriam exatos, devido a todos os pontos estarem sobre esta, os coeficientes seriam obtidos de forma exata.

\section{CONCEITO DE RETA MÉDIA}

Em estatística, a regressão linear é um método para a estimativa do valor condicional de uma certa variável $Y$, dados os valores de uma outra variável $X$. A variável de interesse $Y$ é convencionalmente chamada de variável de resposta, enquanto a variável $X$ é chamada de variável de predição. A regressão em geral é um método para estimar o valor de uma variável esperada. O termo linear é usado devido ao fato de a variável de resposta e de predição serem tomadas como uma função linear de alguns parâmetros. Esses parâmetros, então, definem uma reta caracterizada por um coeficiente angular (aqui chamado de $b$, de modo a não confundir com o $\mathrm{Ca}$ do método anterior) e um coeficiente linear (a).

Deste modo, dada a distribuição das variáveis $X$ e $Y$, pode-se determinar uma reta média, como na Equação (7), de modo a relacionar essas duas variáveis.

$$
Y=a \cdot X+b
$$

O método de regressão linear para a aquisição dos valores de a e b passa pela avaliação da soma dos dados. Assim, somam-se os valores de $X$ e $Y$, além de seus 
respectivos quadrados, e o produto $X Y$ para obter os valores das somas apresentadas nas Equações (10) a (12).

$$
\begin{gathered}
S_{x}=x_{1}+x_{2}+x_{3}+\cdots+x_{n} \\
S_{y}=y_{1}+y_{2}+y_{3}+\cdots+y_{n} \\
S_{x x}=x_{1}^{2}+x_{2}^{2}+x_{3}^{2}+\cdots+x_{n}^{2} \\
S_{y y}=y_{1}^{2}+y_{2}^{2}+y_{3}^{2}+\cdots+y_{n}^{2} \\
S_{x y}=x_{1} y_{1}+x_{2} y_{2}+x_{3} y_{3}+\cdots+x_{n} y_{n}
\end{gathered}
$$

Essas somas são então utilizadas para determinar os parâmetros característicos da reta média derivada do método de regressão linear. Dessa forma, define-se o coeficiente linear $b$ da reta média através da Equação (15), onde $n$ é o número de elementos em cada um dos vetores que representam as variáveis $X$ e $Y$.

$$
b=\frac{n S_{x y}-S_{x} S_{y}}{n S_{x x}-S_{x} S_{y}}
$$

Assim, o coeficiente angular $a$ é calculado levando-se em conta o resultado da estimativa de $b$, através da Equação (16). Como resultado, a reta média sempre passa através do ponto central $(x, y)=(S x / n, S y / n)$.

$$
a=\frac{S_{y}-b S_{x}}{n}
$$

Além disso, o coeficiente de correlação c pode ser calculado através da Equação (17) para determinar quão boa é a aproximação linear da relação entre $X$ e $Y$. Nesse caso, valores próximos de zero indicam um modelo ineficiente, enquanto que valores iguais a 1 indicam que todos os pontos de $Y$ estão sobre a reta média.

$$
c=\frac{n S_{x y}-S_{x} S_{y}}{\sqrt{\left(n S_{x x}-S_{x}^{2}\right)\left(n S_{y y}-S_{y}^{2}\right)}}
$$

Na Fig. 3 apresentam-se as curvas referentes ao fluxo de tráfego entre os nós 1 e 3 e para as taxas que determinam 5, 10 e 15\% de carga em uma rede usando topologia MS-16. Os resultados apresentados nesta figura foram simulados e a reta formada pelos pontos foi comparada. A variável $X$ é a ordem de geração dos pacotes e $Y$ é o tempo de chegada no nó de destino. Para este exemplo, não se usa DR e, devido à baixa carga, pode-se simular de maneira satisfatória o caso SF.

Nota-se que as três curvas representam retas que possuem certas características. Uma delas é que a inclinação (derivada ou coeficiente angular) depende da taxa de bits $R$, como já foi adiantado. Outra característica é que os primeiros pacotes sempre chegam ao nó de destino no mesmo instante de tempo ti e, por conta disso, esse é o ponto de intersecção entre todas as retas. Essa é uma propriedade interessante, já que a reta para o caso SF pode ser determinada com precisão a partir dos dados da rede em baixa carga.

Além desses fatos, o tempo de simulação total é também dependente da taxa $R$ quando mantido o mesmo número de pacotes gerados na rede. Para o cenário da Fig. 3, foram gerados 21 pacotes para cada valor de taxa.

A título de comparação, apresentam-se na Tabela 1 os atributos das retas obtidas para o caso da Fig. 3, em que $a$ e $b$ foram obtidos através da regressão linear dos dados e $C a$ e $C b$ foram obtidos através das Equações (5) e (8), evidenciando que os dois métodos levam a um mesmo resultado para as condições de baixa carga sem DR.

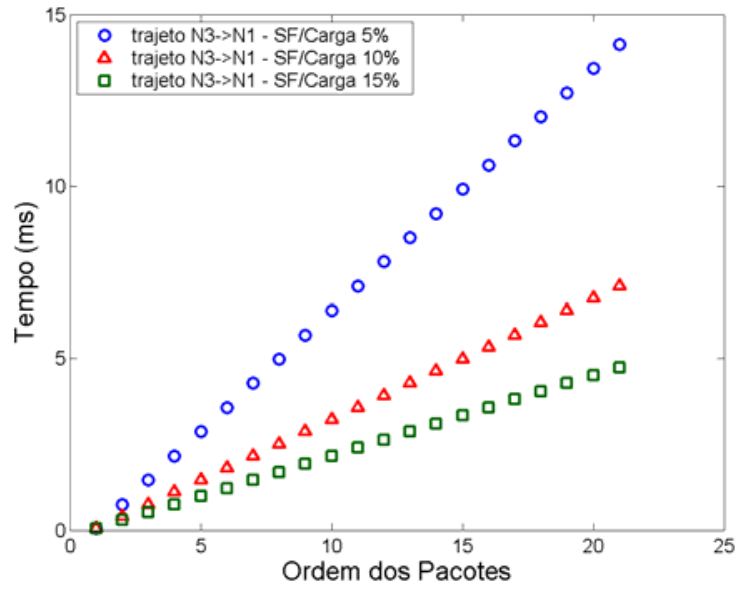

Fig. 3. Relação entre tempo de chegada e ordem dos pacotes.

Tabela 1

Comparação entre os atributos das retas obtidas usando regressão linear e teoria de tráfego.

\begin{tabular}{|c|c|c|c|c|}
\hline \multirow{4}{*}{ Carga } & \multicolumn{4}{|c|}{ Atributos da Reta Média } \\
\cline { 2 - 5 } & \multicolumn{2}{|c|}{ Regressão Linear } & \multicolumn{2}{c|}{ Teoria de Tráfego } \\
\cline { 2 - 5 } & $\begin{array}{c}\text { Coeficiente } \\
\text { Angular }(a)\end{array}$ & $\begin{array}{c}\text { Coeficiente } \\
\text { Linear }(b)\end{array}$ & $\begin{array}{c}\text { Coeficiente } \\
\text { Angular } \\
(\mathrm{Ca})\end{array}$ & $\begin{array}{c}\text { Coeficiente } \\
\text { Linear }(\mathrm{Cb})\end{array}$ \\
\hline $5 \%$ & $7,040 \times 10^{-4}$ & $-6,510 \times 10^{-4}$ & $7,040 \times 10^{-4}$ & $-6,510 \times 10^{-4}$ \\
\hline $10 \%$ & $3,520 \times 10^{-4}$ & $-2,990 \times 10^{-4}$ & $3,520 \times 10^{-4}$ & $-2,990 \times 10^{-4}$ \\
\hline $15 \%$ & $2,346 \times 10^{-4}$ & $-1,814 \times 10^{-4}$ & $2,346 \times 10^{-4}$ & $-1,816 \times 10^{-4}$ \\
\hline
\end{tabular}

Entretanto, a principal motivação deste estudo foi desenvolver uma metodologia para avaliar o nível de desordem causado pela deflexão de pacotes. Por conta disso, os coeficientes $a$ e $c$ provenientes da regressão linear serão usados a seguir como parâmetros de comparação com o coeficiente $\mathrm{Ca}$ calculado analiticamente. Desprezaram-se as influências dos coeficientes $b$ e $C b$ devido ao fato de estes serem mais sensíveis às pequenas variações no intervalo entre pacotes sucessivos, o que levaria à aquisição de resultados não confiáveis. 


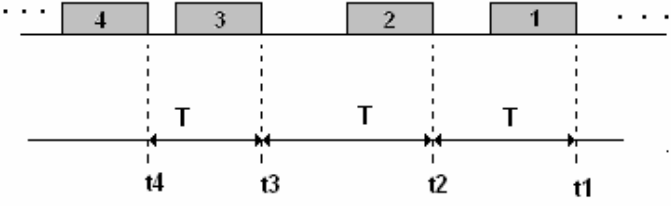

Fig. 4. Chegada de pacotes no nó de destino (caso DR).

\section{NÍVEL DE DESORDEM dos PaCOTES}

O tipo de tráfego adotado na rede é tal que o intervalo entre um pacote e outro $T$ não é estritamente constante e varia em torno de uma média, como mostrado na Figura 4. Além disso, o DR pode causar inversão de ordem de chegada dos pacotes.

\section{Tabela 1}

Parâmetros para o cálculo do nível de desordem.

\begin{tabular}{|c|c|c|c|c|}
\hline Rota & $C a$ & $C b$ & $a$ & $b$ \\
\hline $3-1$ & $3,520 \times 10^{-4}$ & $-2,990 \times 10^{-4}$ & $4,019 \times 10^{-4}$ & $-2,962 \times 10^{-4}$ \\
\hline $15-6$ & $3,520 \times 10^{-4}$ & $2,510 \times 10^{-4}$ & $3,713 \times 10^{-4}$ & $-1,588 \times 10^{-4}$ \\
\hline
\end{tabular}

Dessa forma, nem todos os pontos no caso de DR estarão sobre a reta média, como acontece para o SF e, assim, os parâmetros $a, b$ e $c$ apresentam-se como indicativos do comportamento do tempo de chegada dos pacotes nos nós de destino. Como exemplo, apresentamse na Tabela 1 os parâmetros $a, b, C a$ e $C b$, para os fluxos de tráfego provenientes do nó 3 com destino ao nó 1 e do nó 15 com destino ao nó 6, em uma topologia MS16. Para estes mesmos trajetos o parâmetro $c$ tem valor, respectivamente, de 0,99944 e 0,99895.

Esses resultados são visualizados a partir da Fig. 5, em que são mostrados os pontos referentes à chegada de pacotes SF e os pontos para DR, com sua respectiva reta média obtida a partir do método de regressão linear. A partir desses dados, podem-se comparar os dois coeficientes (angular e linear) dos dois métodos, com relação a todos os fluxos de tráfego presentes na rede. Assim, define-se um novo parâmetro $E_{a}$ que estará relacionado à variação do coeficiente angular devido ao DR através da Equação (18). Nesta equação o $N$ é o número de nós da topologia.

$$
E_{a}=\frac{\sum_{i=1}^{N \cdot(N-1)} a_{i}}{N \cdot(N-1)}
$$

Da mesma forma, define-se com a Equação (19) o parâmetro $E_{c}$, relacionado ao nível de correlação entre a reta média e os pontos. A partir destas, define-se o fator de desordem $E$ como o produto apresentado na Equação (20).

$$
E_{c}=\frac{\sum_{i=1}^{N \cdot(N-1)} c_{i}}{N \cdot(N-1)}
$$

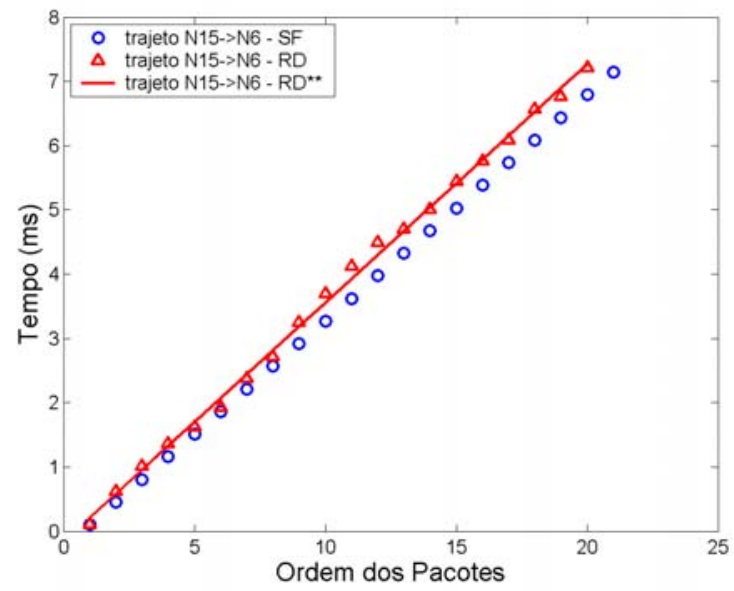

(a)

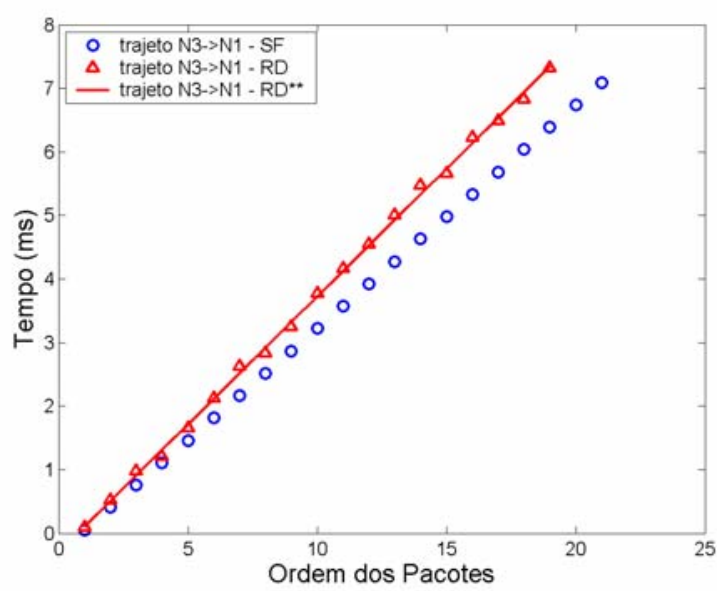

(b)

Fig. 5. Ilustração da regressão linear sobre os dados do tempo de chegada dos pacotes.

$$
E=\frac{E_{a} \cdot E_{c}}{C_{a}}-1
$$

Além do DR, um outro fator para o qual o fator $E$ deve ser bastante sensível é em relação à ocupação dos buffers eletrônicos de entrada, já que estes podem influenciar no tempo de chegada dos pacotes. Por este motivo, os resultados aqui apresentados levam em consideração a avaliação de uma topologia otimizada para o número médio de hops OMT-16 [7] em comparação com sua análoga MS-16, tendo buffers eletrônicos de entrada com capacidade de zero (sem buffer), 10 e 30 pacotes.

Na Figura 6, apresenta-se a evolução do coeficiente angular médio $E_{a}$, calculado através de regressão linear em função da carga na rede, bem como seu respectivo desvio padrão. Da mesma forma, a Fig. 7 e a Fig. 8 apresentam a evolução dos parâmetros de coeficiente linear e coeficiente de correlação, com seus respectivos desvios padrão. Como visto anteriormente, estes parâmetros são utilizados para o cálculo do fator de desordem $E$, do qual se espera obter subsídios para a avaliação do nível de desordem dos pacotes nas redes. 
(a)

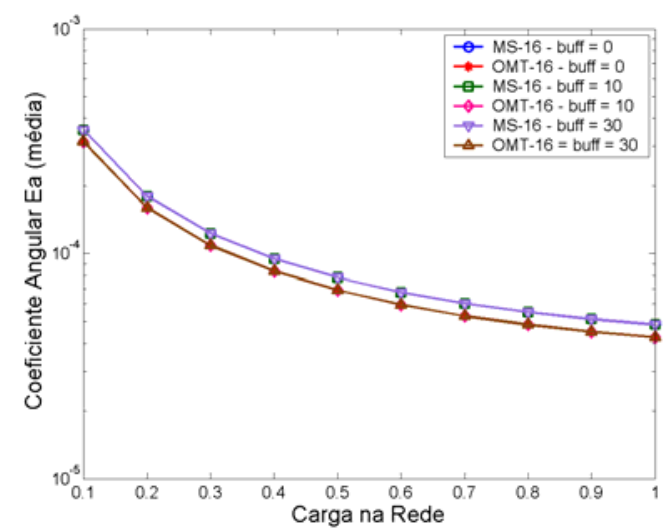

Fig. 6. Coeficiente Angular $E_{a}$, (a) média e (b) desvio padrão.

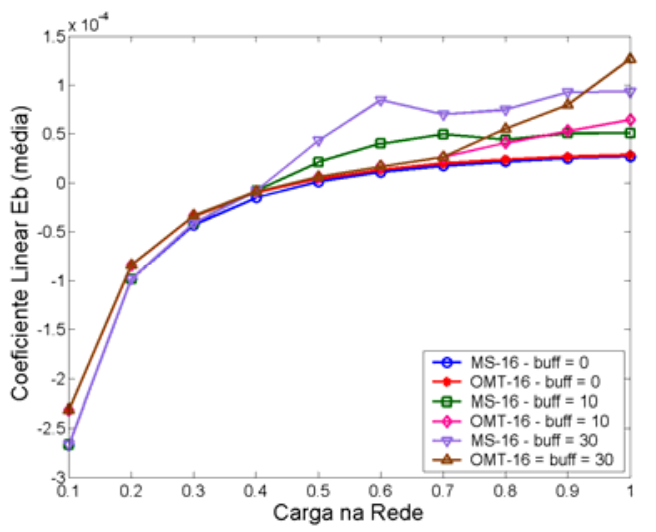

(a)

Fig. 7. Coeficiente Linear, $E_{b}$, (a) média e (b) desvio padrão.

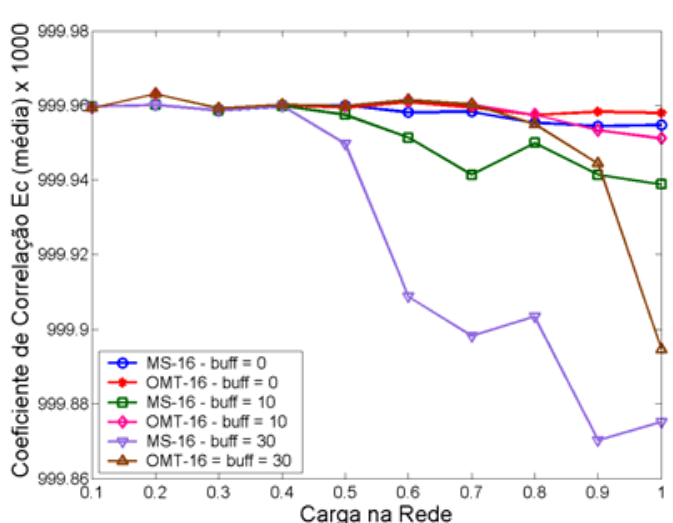

(a)

Fig. 8. Coeficiente de Correlação, $E_{c}$, (a) média e (b) desvio padrão.

Como já era previsto, o fato de haver buffers eletrônicos faz que o nível de desordem aumente com o tamanho destes, afirmação esta que pode ser suportada pela observação dos parâmetros de coeficiente linear e coeficiente de correlação. Entretanto, para a composição do fator de desordem $\mathrm{E}$, não foi levado em conta o coeficiente linear por questões já expostas anteriormente. Um comportamento não intuitivo e que é comprovado

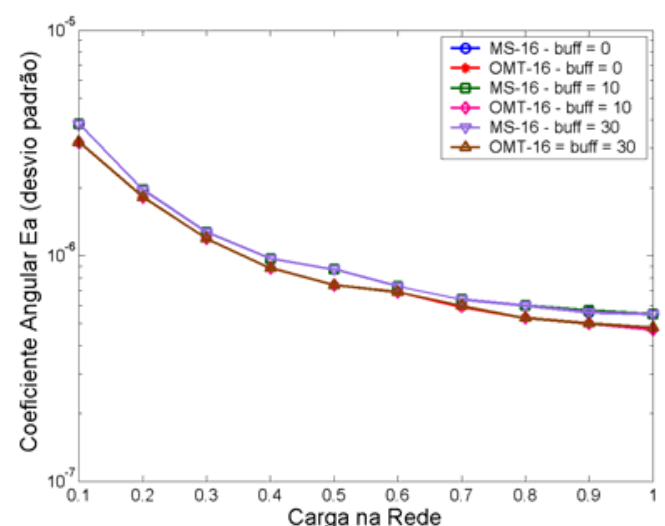

(b)

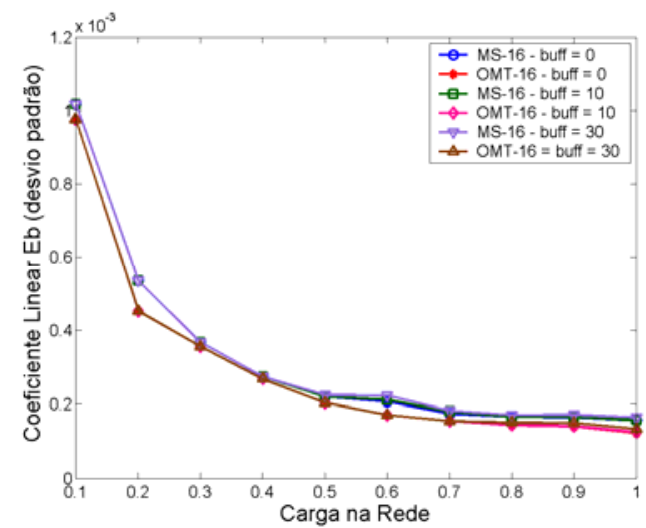

(b)

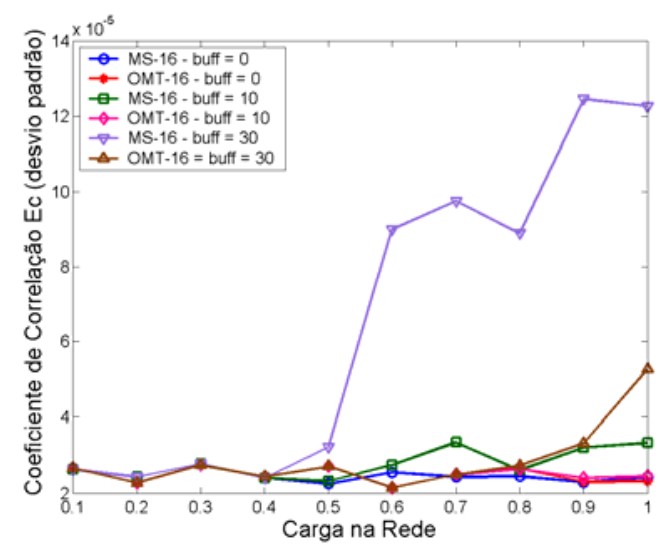

através da observação dos resultados apresentados na Fig. 9 é que as OMT's apresentam um baixo valor para $\mathrm{E}$ até aproximadamente $50 \%$ de carga da rede, a partir da qual existem muitas variações nesse fator, evidenciando que o nível de desordem, ou seja, a quantidade de pacotes que chegam fora de ordem ao nó de destino é diretamente proporcional à carga que os pacotes experimentam. De forma a ter um parâmetro quantitativo para essa variação, 
adotou-se o fator $E_{\text {desvio, }}$ o qual é calculado através da Equação (21), que por sua vez utiliza os valores de desvio padrão dos parâmetros de coeficiente angular e de correlação médios, bem como o coeficiente angular teórico para o caso de roteamento SF Ca.

$$
E_{\text {desvio }}=\frac{\operatorname{std}\left(E_{a}\right) \cdot \operatorname{std}\left(E_{c}\right)}{C_{a}^{2}}
$$
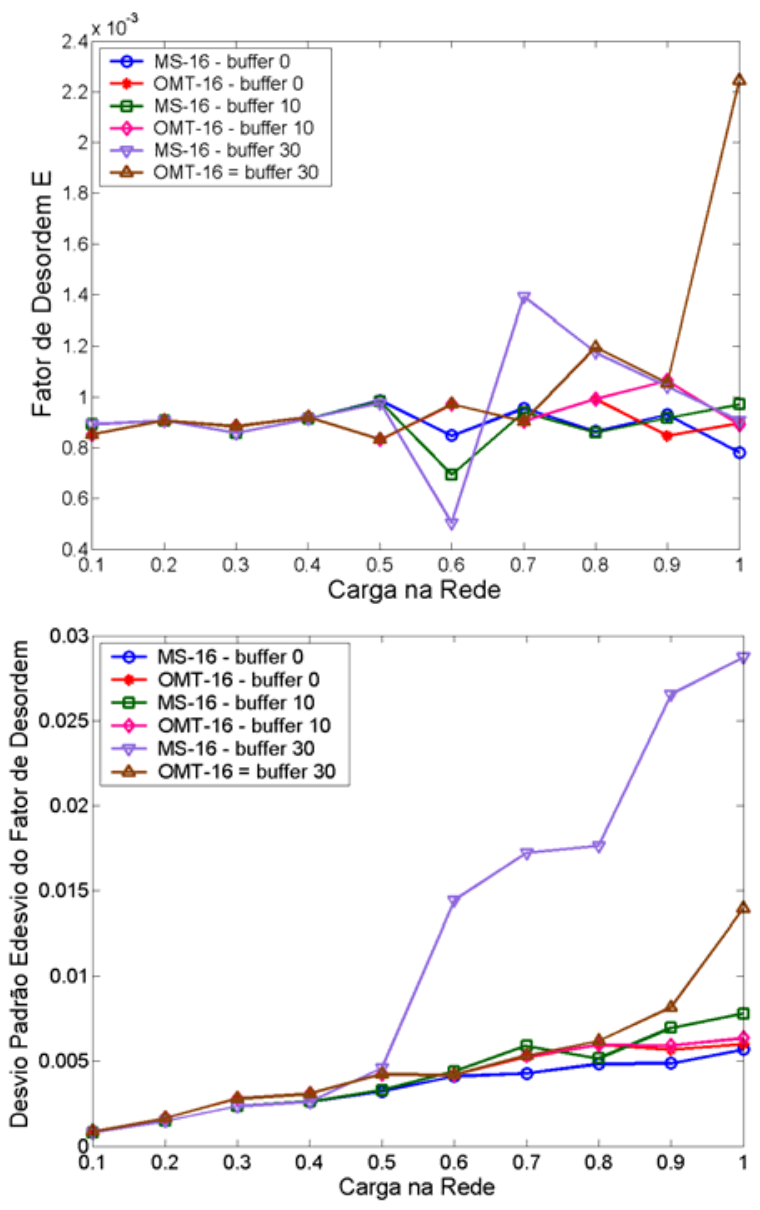

Fig. 9. Coeficiente de desordem, (a) $E$ e (b) $E_{\text {desvio }}$.

Dessa forma, analisando os resultados até então obtidos, pode-se concluir que a desordem dos pacotes na arquitetura proposta cresce com o tamanho dos buffers na região de altas cargas e praticamente não é afetada por estes na região de baixas cargas, em que o nível de desordem tem uma evolução suave, aproximadamente linear, como pode ser visto na Fig. 9 (b), apresentando variações bem maiores a medida em que a carga aumenta e para os casos de buffers eletrônicos de maior capacidade.

Apesar da proposta de métrica apresentada aqui ter sido capaz de subsidiar uma análise quantitativa preliminar do nível de inversão de pacotes, este não é ainda um assunto fechado, sendo que em futuro próximo se pretende estudar outras relações entre os coeficientes apresentados e analisados, de forma a alcançar novos dados para a avaliação deste problema. O mais importante neste momento é que em princípio a métrica já foi capaz de embasar a afirmação de que o nível de desordem não é problemático até cerca de $50 \%$ de carga na rede, considerando a arquitetura proposta.

\section{CONCLUSÃO}

Vimos que um dos grandes problemas na operação de redes ópticas comutadas por pacotes ou rajadas (OPS/OBSN) é que estas podem apresentar níveis de desordem relativamente altos em seus nós de destino sob certas condições de tráfego, quando submetidas ao roteamento por deflexão. Esses níveis de desordem são prejudiciais á comunicação, pois aumentam a latência para os usuários da rede, sendo que é necessário um tempo adicional para o reordenamento dos pacotes em um armazenador de saída no destino. Apresenta-se no presente trabalho uma métrica para avaliação quantitativa desse nível de desordem, onde se fez uso da estatística de distribuição de tráfego para modelar o comportamento dos tempos de atraso das chegadas e, com isso, estabelecer um parâmetro de correlação com o comportamento observado efetivamente na rede.

O método da reta média, usando regressão linear apresenta-se simples e com bons resultados (boa reprodutibilidade) permitindo a partir daqui continuidade nos trabalhos com formulações mais precisas, e maior confiabilidade no dimensionamento dos armazenadores.

\section{REFERÊNCIAS}

[1] S. J. Yoo, "Optical Packet and Burst Switching Technologies for the Future Photonic Internet", $J$. Lightwave Technology, vol. 24, no. 12, December 2006.

[2] S. Yao, B. Mukherjee, S.J.Yoo, S.Dixit, "A Unified Study of Contention Resolution Schemes in Optical packet switching Networks", IEEE J. Lightwave Tech, vol.21, no.3, p.672, March 2003.

[3] S. Yao and B. Mukherjee, "Advances in photonic packet switching: an overview", IEEE Communications Magazine pp.84-94, February 2000.

[4] A. S. Acampora and S. I. A. Shah, "Multihop lightwave network: a comparison of store-and forwaDR and hot potato routing”, IEEE Trans. on Communications, vol.40, no.6, pp.1082-1090, 1992.

[5] A. M. Legendre. "Nouvelles méthodes pour la détermination des orbites des comètes”, Sur la Méthode des moindres quarrés, appears as an appendix, 1805.

[6] R. A. Fisher. "The goodness of fit of regression formulae, and the distribution of regression coefficients", J. Royal Statist. Soc., 85, 597-612, 1922.

[7] L. H. Bonani, F. R. Barbosa, E. Moschim, R. R. F. Attux, R. Arthur, "Optimization of Generalized 2x2 Mesh Topologies using an Evolutionary Algorithm”, Revista Telecomunicações, Inatel, Santa Rita do Sapucaí, aceito para publicação. 